\title{
Spatial Organization and Dynamics of the Extracellular Space in the Mouse Retina
}

\author{
Sidney P. Kuo, Pei-Pei Chiang, Amy R. Nippert, and ${ }^{\circ}$ Eric A. Newman \\ Department of Neuroscience, University of Minnesota, Minneapolis, Minnesota 55455
}

The extracellular space (ECS) plays an important role in the physiology of neural circuits. Despite our detailed understanding of the cellular architecture of the mammalian retina, little is known about the organization and dynamics of the retinal ECS. We developed an optical technique based on two-photon imaging of fluorescently labeled extracellular fluid to measure the ECS volume fraction $(\alpha)$ in the ex vivo retina of male and female mice. This method has high spatial resolution and can detect rapid changes in $\alpha$ evoked by osmotic challenge and neuronal activity. The measured ECS $\alpha$ varied dramatically in different layers of the adult mouse retina, with $\alpha$ equaling $\sim 0.050$ in the ganglion cell layer, $\sim 0.122$ in the inner plexiform layer (IPL), $\sim 0.025$ in the inner nuclear layer (INL), $\sim 0.087$ in the outer plexiform layer, and $\sim 0.026$ in the outer nuclear layer (ONL). ECS $\alpha$ was significantly larger early in retinal development; $\alpha$ was $67 \%$ larger in the IPL and $100 \%$ larger in the INL in neonatal mice compared with adults. In adult retinas, light stimulation evoked rapid decreases in ECS $\alpha$. Light-driven reductions in ECS $\alpha$ were largest in the IPL, where visual stimuli decreased $\alpha$ values $\sim 10 \%$. These light-evoked decreases demonstrate that a physiological stimulus can lead to rapid changes in ECS $\alpha$ and indicate that activity-dependent regulation of extracellular space may contribute to visual processing in the retina.

Key words: diffusion; extracellular space; glial cells; light response; retina; volume fraction

\section{Significance Statement}

The volume fraction of the extracellular space (ECS $\alpha$ ), that portion of CNS tissue occupied by interstitial space, influences the diffusion of neurotransmitters from the synaptic cleft and the volume transmission of transmitters. However, ECS $\alpha$ has never been measured in live retina, and little is known about how ECS $\alpha$ varies following physiological stimulation. Here we show that ECS $\alpha$ values vary dramatically between different retinal layers and decrease by $10 \%$ following light stimulation. ECS $\alpha$ differences within the retina will influence volume transmission and light-evoked $\alpha$ variations may modulate synaptic transmission and visual processing in the retina. Activity-dependent ECS $\alpha$ variations may represent a mechanism of synaptic modulation throughout the CNS.

\section{Introduction}

The extracellular space (ECS) is composed of narrow, interconnected pathways that permit the diffusion of ions, signaling molecules, and metabolites between cells within the CNS. By regulating how molecules diffuse through neural tissue, the ECS can strongly influence CNS function. For example, the local geometry of the ECS around synapses affects how neurotransmitters diffuse away from release sites and therefore shapes the spillout of transmitters from the synaptic cleft onto extrasynaptic

\footnotetext{
Received July 3, 2020; revised Aug. 24, 2020; accepted Aug. 31, 2020.

Author contributions: S.P.K., A.R.N., and E.A.N. designed research; S.P.K., P.P.C., A.R.N., and E.A.N. performed research; S.P.K., A.R.N., and E.A.N. analyzed data; S.P.K., A.R.N., and E.A.N. wrote the paper.

This research was funded by National Institutes of Health Grants R01-EY-026514, R01-EY-026882, and P30EY-011374 to E.A.N.; T90-DE-0227232 to S.P.K.; and T32-EY-025187 to A.R.N. We thank Charles Nicholson for helpful comments on an earlier version of the manuscript.

The authors declare no competing financial interests.

Correspondence should be addressed to Eric A. Newman at ean@umn.edu.

https://doi.org/10.1523/JNEUROSCI.1717-20.2020

Copyright $\odot 2020$ the authors
}

receptors (receptors localized outside of the cleft), the spillover of transmitters between neighboring synapses, and the volume transmission of transmitters to distant receptors (Syková and Nicholson, 2008; Nicholson and Hrabětová, 2017). Studies have demonstrated changes in ECS structure during development (Lehmenkühler et al., 1993; Vorisek and Sykova, 1997), across the sleep-wake cycle (Xie et al., 2013), and during disease progression (Mazel et al., 2002; Reum et al., 2002; Syková et al., 2005; Slais et al., 2008; Tønnesen et al., 2018). These studies, along with work showing that electrical stimulation of neuronal activity can drive changes in ECS properties (Svoboda and Syková, 1991; Prokopová-Kubinová and Syková, 2000), raise the intriguing possibility that dynamic alterations to ECS structure may provide an unappreciated mechanism for the activity-dependent modulation of synaptic and volume transmission. However, the extent to which physiological activity within a neural circuit can result in altered ECS properties is not known.

The ECS plays an important role in retinal physiology. For example, the spatial extent of neuromodulatory signaling by 
transmitters such as dopamine (Witkovsky, 2004) and the recruitment of extrasynaptic receptors following synaptic release (Chen and Diamond, 2002; Zhang and Diamond, 2009) are shaped by the properties of the ECS in the retina. Despite our detailed understanding of the cellular architecture of the mammalian retina, little is known regarding the organization or dynamics of the retinal ECS. This is in part because the compact, layered structure of the retina poses a particular challenge to the traditional method of measuring ECS properties in live tissue, termed "real-time iontophoresis" (Nicholson and Hrabětová, 2017). In this approach, an ion, typically tetramethylammonium (TMA), is iontophoretically injected into a tissue. The ion diffuses through the ECS, and its buildup is measured at a distance of $\sim 150 \mu \mathrm{m}$ by an ion-selective microelectrode. Values of ECS volume fraction $(\alpha)$ and tortuosity $(\lambda)$ are derived from the time course and magnitude of the rise in TMA concentration at the ion-selective microelectrode. An important assumption of the real-time iontophoresis technique is that the tissue being studied is isotropic; that is, the properties of the ECS are uniform in all directions (but see Rice et al., 1993; Saghyan et al., 2012). This assumption does not hold in the retina, which is composed of discrete layers (e.g., somatic vs synaptic) with very different cellular organizations. The spatial resolution of the real-time iontophoresis technique, which is limited by the distance between iontophoretic and ion-selective electrodes $(\sim 150 \mu \mathrm{m})$, is also not compatible with the dimensions of the retina $(\sim 180 \mu \mathrm{m}$ thick in mouse).

We report here an optical method for measuring ECS $\alpha$, the fraction of the total volume of tissue occupied by the ECS, in the retina. This technique, based on two-photon (2P) excitation of a fluorescent dye that labels the ECS, allows us to determine the precise values of $\alpha$ in different retinal layers with high spatial resolution and to measure changes in $\alpha$ during development and during light stimulation. We find that $\alpha$ varies nearly fivefold within different layers of the retina, from $\sim 0.025$ in the inner nuclear layer (INL) to $\sim 0.122$ in the inner plexiform layer (IPL), and that $\alpha$ is $46-100 \%$ larger in different layers of the developing retina than in the adult retina. We also show that $\alpha$ can decrease by $>10 \%$ in response to visual stimulation. These light-evoked decreases demonstrate that a physiological stimulus can evoke rapid changes in ECS $\alpha$ and indicate that activity-dependent regulation of ECS may contribute to visual processing in the retina.

\section{Materials and Methods}

\section{Ethics statement}

All experimental procedures were approved by and adhered to the guidelines of the Institutional Animal Care and Use Committee of the University of Minnesota.

\section{Retinal preparations}

All experiments were performed on tissue from 0 - to 12-week-old male and female C57BL/6 mice. Mice were anesthetized with isoflurane and killed by cervical dislocation. The eyes were enucleated and the cornea, lens, and vitreous were removed in room temperature, oxygenated $(95 \%$ $\mathrm{O}_{2}, 5 \% \mathrm{CO}_{2}$ ) artificial CSF (ACSF). The ventral portion of eyecups were then used to obtain either of two experimental preparations.

Eyecup preparation. Experiments that assessed osmotic and lightdriven changes in ECS $\alpha$ used an eyecup preparation in which the retina remained attached to the underlying pigment epithelium, choroid, and sclera. The eyecup was trimmed down to a $\sim 3 \times 3 \mathrm{~mm}$ square and was mounted flat onto a poly-L-lysine-coated coverglass on the bottom of a perfusion chamber with the vitreal surface up. Tissue was secured in place with a harp made from platinum wire and nylon threads.
Isolated retina preparation. The isolated retina preparation was used when determining the depth-dependent fluorescence signal attenuation and when measuring ECS $\alpha$ in different layers to minimize the amount of dye-labeled ACSF between the tissue and microscope objective and to achieve more uniform tissue flatness than was possible in the eyecup preparation. The retina was dissected from the eyecup, mounted onto a coverglass with the vitreal surface down, and held in place with a harp secured to the coverglass with drops of melted paraffin wax. The coverglass was then inverted and placed on the top surface of the perfusion chamber. In both experimental preparations, the vitreal surface of the retina faced the microscope objective. For both preparations, retinas were perfused at $\sim 7 \mathrm{ml} / \mathrm{min}$ with warmed $\left(\sim 32^{\circ} \mathrm{C}\right)$, oxygenated ACSF containing the membrane-impermeant fluorescent dye calcein $(0.1 \mathrm{~mm}$; catalog \#C0875, Sigma-Aldrich).

\section{Solutions}

The ACSF contained (in mM): $125 \mathrm{NaCl}, 2.5 \mathrm{KCl}, 1.2 \mathrm{CaCl}_{2}, 1.5 \mathrm{MgSO}_{4}$, $1.25 \mathrm{NaH}_{2} \mathrm{PO}_{4}, 20$ glucose, 0.5 L-glutamine, 0.1 sodium ascorbate, and $26 \mathrm{NaHCO}_{3}$, at pH 7.4 and $\sim 310 \mathrm{mOsm} / \mathrm{L}$. Thirty millimolar mannitol was added to the control ACSF solution to create the hypertonic solution $(\sim 340 \mathrm{mOsm} / \mathrm{L})$. The hypotonic ACSF $(\sim 280 \mathrm{mOsm} / \mathrm{L})$ contained 110 $\mathrm{mm} \mathrm{NaCl}$ instead of $125 \mathrm{~mm} \mathrm{NaCl}$. For experiments examining the effect of hypotonic solution, the control ACSF ( $\sim 310$ mOsm) was modified to contain $110 \mathrm{~mm} \mathrm{NaCl}$ and $30 \mathrm{~mm}$ mannitol.

\section{Two-photon imaging and visual stimulation}

In both retinal preparations, a custom-built $2 \mathrm{P}$ laser-scanning microscope equipped with a $25 \times, 1.05$ numerical aperture objective (catalog \#XLMPLN25XWLP2, Olympus) was used to image calcein in the extracellular space. The microscope was based on a previous design (Rosenegger et al., 2014) and was controlled using ScanImage2018 software (Vidrio Technologies). Two-photon excitation of calcein was achieved using a Ti: sapphire laser (Chameleon Vision, Coherent) tuned to 800 or $920 \mathrm{~nm}$ (post-objective laser power, 2-6 $\mathrm{mW}$ ), and calcein fluorescence emission was detected using a bandpass filter (catalog \#et525/50m-2P, Chroma) and a GaAsP photomultiplier tube (catalog \#H10770PA-40, Hamamatsu). Images $(512 \times 512$ pixels; $0.17 \mu \mathrm{m} /$ pixel $)$ were acquired at a $2-5 \mathrm{~Hz}$ frame rate. The point-spread function of the microscope was measured by imaging fluorescent beads (diameter, $175 \mathrm{~nm}$; excitation wavelength, $780 \mathrm{~nm}$ ). The full-width at half-maximum (FWHM) of Gaussian fits to the fluorescence profiles of individual beads was $0.36 \pm 0.02 \mu \mathrm{m}$ in the $x-y$ plane and $1.40 \pm 0.05 \mu \mathrm{m}$ in the $z$-axis ( $n=6$ beads).

Retinas were stimulated using the following two light sources, both of which were built into the optical path of the $2 \mathrm{P}$ microscope and projected through the objective. (1) A $405 \mathrm{~nm}$ light from a laser diode (cata$\log$ \#L405P150, ThorLabs) was projected through a diffuser and circular mask to produce a $100-\mu \mathrm{m}$-diameter spot on the retina. Stimuli produced a maximal photon flux of $15.3 \times 10^{5}$ photons $/\left(\mu \mathrm{m}^{2} \times \mathrm{s}\right)$. Assuming a cone collecting area of $1.0 \mu \mathrm{m}^{2}$ (Naarendorp et al., 2010), this was equivalent to $1.8 \times 10^{5}$ cone opsin isomerizations per short wavelength-sensitive cone per second $\left(\mathrm{P}^{*} /(\mathrm{S}\right.$ cone $\times$ s $\left.)\right)$. (2) A $405 \mathrm{~nm}$ light from a Digital Light Processing projector (catalog \#PRO4500, Wintech Digital; $912 \times 1140$ pixels, $0.512 \mu \mathrm{m} /$ pixel) that was controlled using Stage Visual Stimulation System software (https://stage-vss.github. io) was used to produce spots of varying diameter $(25-600 \mu \mathrm{m})$ on the retina. Stimuli produced a maximal photon flux of $1.1 \times 10^{5}$ photons/ $\left(\mu \mathrm{m}^{2} \times \mathrm{s}\right)$, equivalent to $1.21 \times 10^{4} \mathrm{P}^{\star} /(\mathrm{S}$ cone $\times \mathrm{s})$. For both stimuli, the stimulus focal plane was located $\sim 100 \mu \mathrm{m}$ farther from the objective than the imaging focal plane so that stimuli were focused on the photoreceptors when imaging the proximal retina.

\section{Measurement of ECS $\alpha$}

The ECS $\alpha$ at different depths within the retina was determined by measuring the spatially integrated dye fluorescence intensity within a layer and comparing it with the dye fluorescence in the superfusate above the retina (Fig. $1 A-C$ ). Similar techniques in which ECS is labeled with a fluorescent dye have been used previously to determine $\alpha$ in brain slices (Zhang and Verkman, 2010; Tønnesen et al., 2018). Assuming that the 


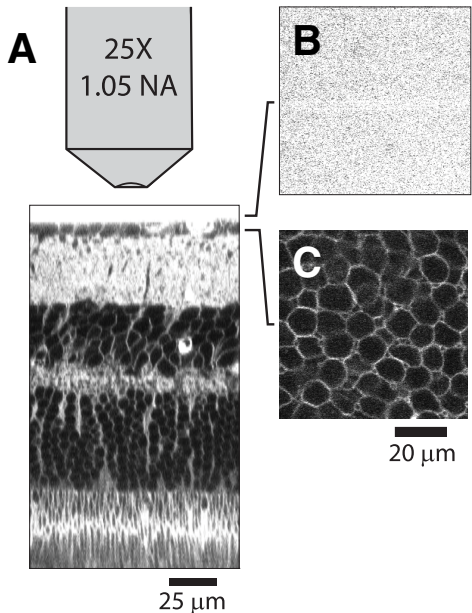

$\mathbf{F}$

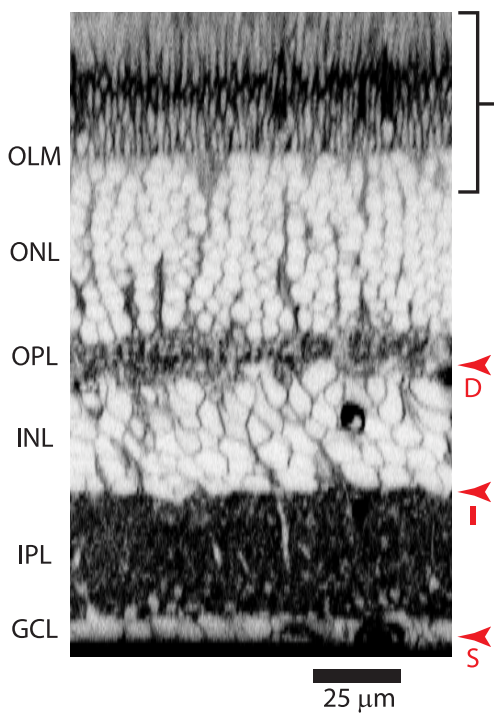

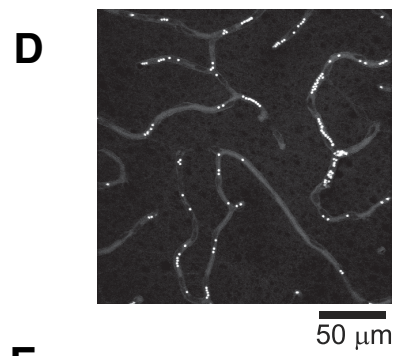
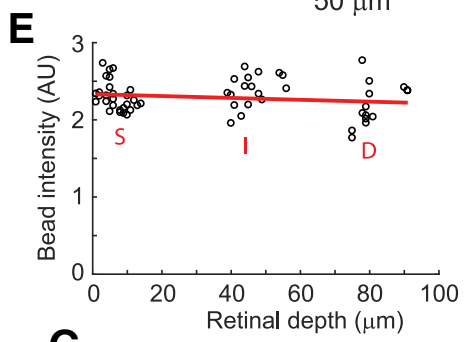

G

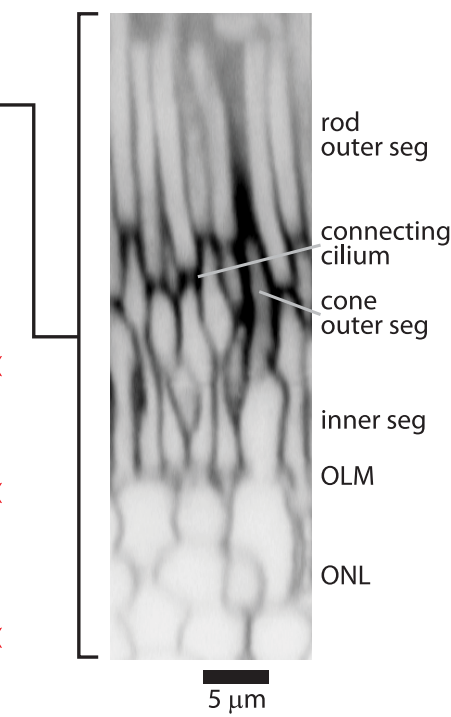

Figure 1. Two-photon fluorescence imaging technique for determining ECS $\alpha$. A, Side view of retina labeled with 0.1 $\mathrm{mm}$ extracellular calcein to illustrate imaging geometry. The image is a vertical reconstruction of a $z$-stack of $2 \mathrm{P}$ microscopy images. $\boldsymbol{B}$, Example $2 \mathrm{P}$ image of bath solution from focal plane above retina. The average calcein fluorescence intensity is used to define ECS $\alpha=1$. C, Example image of calcein fluorescence in the GCL. The dark circular regions are neuronal somata, whereas the bright regions are calcein-labeled ECS between cells. From a comparison of average fluorescence intensity with that in $\boldsymbol{B}, \alpha=0.05$ in the GCL. D, 2P microscopy image of $2-\mu \mathrm{m}$-diameter fluorescent beads within capillaries in the deep capillary layer, $80 \mu \mathrm{m}$ beneath the retina surface. The capillary bed is labeled lightly with calcein. $\boldsymbol{E}$, Intensity of individual beads within capillaries of the superficial (S), intermediate (I), and deep (D) capillary layers of an example retina as a function of depth beneath the retinal surface. The data are fit with an exponential function (red line) that has a length constant of $1879 \mu \mathrm{m}$. $\boldsymbol{F}$, Side view of extracellular calcein fluorescence in the retina reconstructed from a $z$-stack of images ( $1 \mu \mathrm{m}$ step size). Image contrast is inverted so that the calcein-labeled extracellular spaces appear dark and cells appear bright. The vertical orientation is opposite that in $\boldsymbol{A}$. Retinal layers are indicated to the left. OLM, Outer limiting membrane. The irregular black profiles in the GCL and the INL are calcein-filled blood vessels. The locations of the superficial, intermediate, and deep capillary layers are indicated by red arrowheads. $\boldsymbol{G}$, Vertical reconstruction of the photoreceptors, based on a $z$-stack of extracellular calcein fluorescence images obtained at $0.4 \mu \mathrm{m}$ increments. As in $\boldsymbol{F}$, the contrast is inverted. The rod and cone outer segments are clearly visible as are the connecting cilia between the rod inner and outer segments.

dye fills all of the ECS and that the dye concentration within these spaces equals the concentration in the perfusate, then:

$$
a=F_{\text {Retina }} / F_{\text {Perfusate, }}
$$

where $F$ equals the mean calcein fluorescence signal in an imaged plane. Fluorescence was measured in regions of interest (ROIs) $\sim 70 \times 70 \mu \mathrm{m}$. Areas within ROIs that contained blood vessels, which fill with calceinlabeled solution in the ex vivo retina, were excluded from analysis. Cells that filled with calcein, which presumably had compromised health, were also excluded from analysis, and regions of retina with more than two dead cells were not used in experiments. To accurately determine ECS $\alpha$ values throughout the retina, we corrected calcein fluorescence measurements for depth-dependent signal attenuation that arises from the scatter of excitation and emission light. As shown previously, this attenuation increases exponentially with depth (Dunn et al., 2000; Ying et al., 2000; Kobat et al., 2009). The length constant of the exponential attenuation was determined experimentally (see below "Determination of depth-dependent fluorescence signal attenuation") and used to correct ECS $\alpha$ values.

For measurements of ECS $\alpha$, calcein was added to the bath solution at a concentration of $0.1 \mathrm{~mm}$, which is well below the concentration at which the dye self-quenches ( 3 mM; Magzoub et al., 2009). In all experiments, we allowed calcein to equilibrate within the retina for a minimum of $30 \mathrm{~min}$ before measurements to ensure that calcein concentration was uniform throughout the retinal ECS and superfusate. This incubation period was determined from experiments in which the time course of fluorescence changes was observed following a rapid change in bath solution calcein concentration from 0.01 to $0.1 \mathrm{~mm}$. Following a step change in concentration, the calcein fluorescence intensity equilibrated to within $10 \%$ of its final value in $225 \pm 51 \mathrm{~s}$ in the IPL $(26 \mu \mathrm{m}$ beneath the vitreal surface, $n=10)$ and in $469 \pm 62 \mathrm{~s}$ in the outer nuclear layer (ONL; $122 \mu \mathrm{m}$ beneath the surface; $n=2)$. Bleaching of calcein fluorescence did not occur in our experiments; when the excitation laser intensity was increased 30 -fold (to $\sim 180 \mathrm{~mW}$ postobjective laser power) over the highest intensity used for imaging experiments $(\sim 6 \mathrm{~mW})$, we did not observe any change in calcein fluorescence over time. Consistent with previous work in brain slices (Zhang and Verkman, 2010; Tønnesen et al., 2018), calcein was restricted to extracellular compartments when applied in the ACSF superfusing the retina; dim fluorescent signals from neuronal somata in the INL (because of extracellular calcein outside of the focal plane) did not change appreciably over $60 \mathrm{~min}$ of observation $(-0.3 \pm 16.9 \%$; $n=26$ ).

Determination of depth-dependent fluorescence signal attenuation

Attenuation of fluorescence signals due to light scatter within the retina was empirically determined by imaging fluorescent beads within retinal blood vessels. These measurements were performed on isolated retinas in the inverted imaging configuration. Mice were transcardially perfused with a solution containing $2-\mu \mathrm{m}$-diameter fluorescent beads before enucleation of the eyes. One milliliter of bead solution (catalog \#L3030, SigmaAldrich) was spun down to concentrate the beads, washed three times, resuspended in $0.2 \mathrm{ml}$ of saline with $0.05 \%$ Tween, and injected into the heart. After introduction into the vasculature, the beads were clearly visible in the three capillary layers in the retina, which are located in the ganglion cell layer (GCL), at the IPL/INL border, and at the INL/outer plexiform layer (OPL) border (Fig. 1D; Kornfield and Newman, 2014). Beads were imaged with the same excitation wavelength and emission filter used to image calcein. The fluorescence intensity of individual beads was plotted as a function of depth beneath the 
retinal surface and fit with an exponential function (Fig. $1 E)$. Signal attenuation was found to have a mean length constant of $1780 \pm 692 \mu \mathrm{m}$ ( $n=13$ retinas). Thus, signal attenuation is minor, declining by only $8 \%$ at a depth of $150 \mu \mathrm{m}$. This is expected as the retina is largely transparent. All ECS $\alpha$ measurements reported here were corrected for depth-dependent signal attenuation using the mean measured length constant of $1780 \mu \mathrm{m}$. It should be noted that the calcein imaging method likely does not provide accurate measurements of ECS $\alpha$ within the photoreceptor outer segments since absorption of excitation and emission light by photopigments will influence calcein measurements. Consistent with this, image intensity dropped off as the focal plane moved distally through this layer (Fig. 2B; note the $\alpha$ decrease past $180 \mu \mathrm{m}$ depth).

\section{Simulations of calcein diffusion}

The rate of calcein diffusion within the retina was determined with a combination of experimental and modeling techniques. Diffusion was measured experimentally by iontophoretic ejection of calcein from a pipette within the IPL. Calcein $(80 \mathrm{~mm})$ was ejected from glass micropipettes (tip diameter, $\sim 1 \mu \mathrm{m}$; series resistance, $\sim 50 \mathrm{M} \Omega$ ) for $120 \mathrm{~s}$ using a $-5 \mathrm{nA}$ constant current step generated by a Multiclamp 700A Amplifier (Molecular Devices), and fluorescence was measured using $2 \mathrm{P} \mathrm{mi-}$ croscopy within an arc-shaped region of interest located $\sim 75 \mu \mathrm{m}$ laterally from the tip of the iontophoretic pipette in the IPL, INL, or ONL (see Fig. $4 A, B$ ). Ejection current was monitored to ensure that calcein release did not vary during the $120 \mathrm{~s}$ pulse.

Diffusion was modeled using MCell, a software program that combines 3D models of neural tissue with Monte Carlo algorithms to simulate diffusion in the ECS (Tao and Nicholson, 2004; Kerr et al., 2008). CellBlender was used to construct a 3D model of a $200 \times 200 \mu \mathrm{m}$ region of retina that was $144 \mu \mathrm{m}$ thick (Fig. $4 \mathrm{C}$ ). For simplicity, the model was divided into $3 \times 3 \times 3 \mu \mathrm{m}$ contiguous cubes (Tao and Nicholson, 2004). Each cube is composed of compartments representing extracellular and intracellular spaces. The relative proportion between the extracellular and intracellular spaces was adjusted to achieve the appropriate ECS $\alpha$ for each retinal layer. The model contained the following layers: retinal ganglion cell (RGC): $12 \mu \mathrm{m}$ thick, $\alpha=0.05$; IPL: $39 \mu \mathrm{m}$ thick, $\alpha=$ 0.122; INL: $30 \mu \mathrm{m}$ thick, $\alpha=0.025$; OPL: $12 \mu \mathrm{m}$ thick, $\alpha$ $=0.087$; ONL: $51 \mu \mathrm{m}$ thick, $\alpha=0.026$. For simulations of calcein diffusion from a point source, four particles representing calcein molecules were released every $200 \mathrm{~ms}$ from a point at the center of the IPL (2400 particles over 120 s). With 2400 particles, a single simulation run on a Windows computer with a seventh generation Intel Core i5 processor running at $3.5 \mathrm{GHz}$ took $\sim 20 \mathrm{~h}$. Six independent runs were averaged to reduce noise.

The calcein concentration change that would occur upon a rapid decrease in ECS $\alpha$ within the IPL was simulated by instantaneously increasing the number of calcein molecules within the modeled IPL. This test paradigm allowed us to determine the time course over which our fluorescence-based measurements can accurately report changes in ECS $\alpha$. For this simulation, 1000 particles were evenly distributed throughout the entire ECS of the IPL and released at time 0 . We define an interstitial space diffusion coefficient $\left(D_{i}\right)$ for the diffusion of particles within the ECS in the simulations. The $D_{i}$ is smaller (slower) than the free diffusion coefficient in water as particles within the ECS are slowed down by interstitial viscosity, dead spaces, and other factors (Nicholson and Hrabětová, 2017). The $D_{i}$ does not include a factor for the increased path length that particles must travel because of the geometry of the ECS as that is simulated by MCell. As explained below, $D_{i}$ was determined by

B
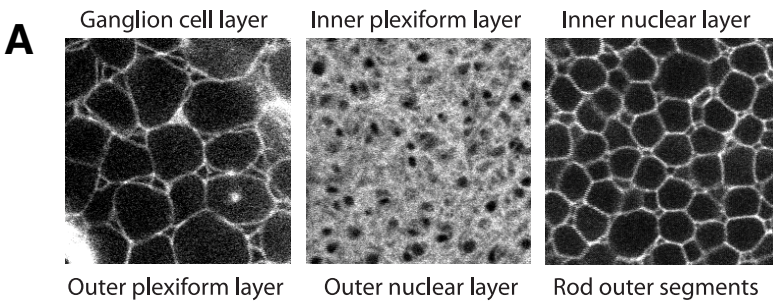

Outer nuclear layer
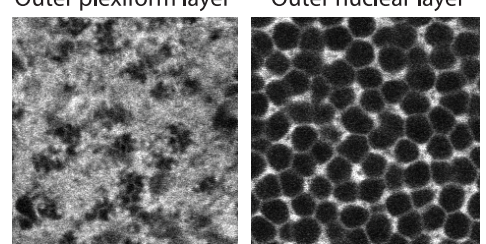

Rod outer segments
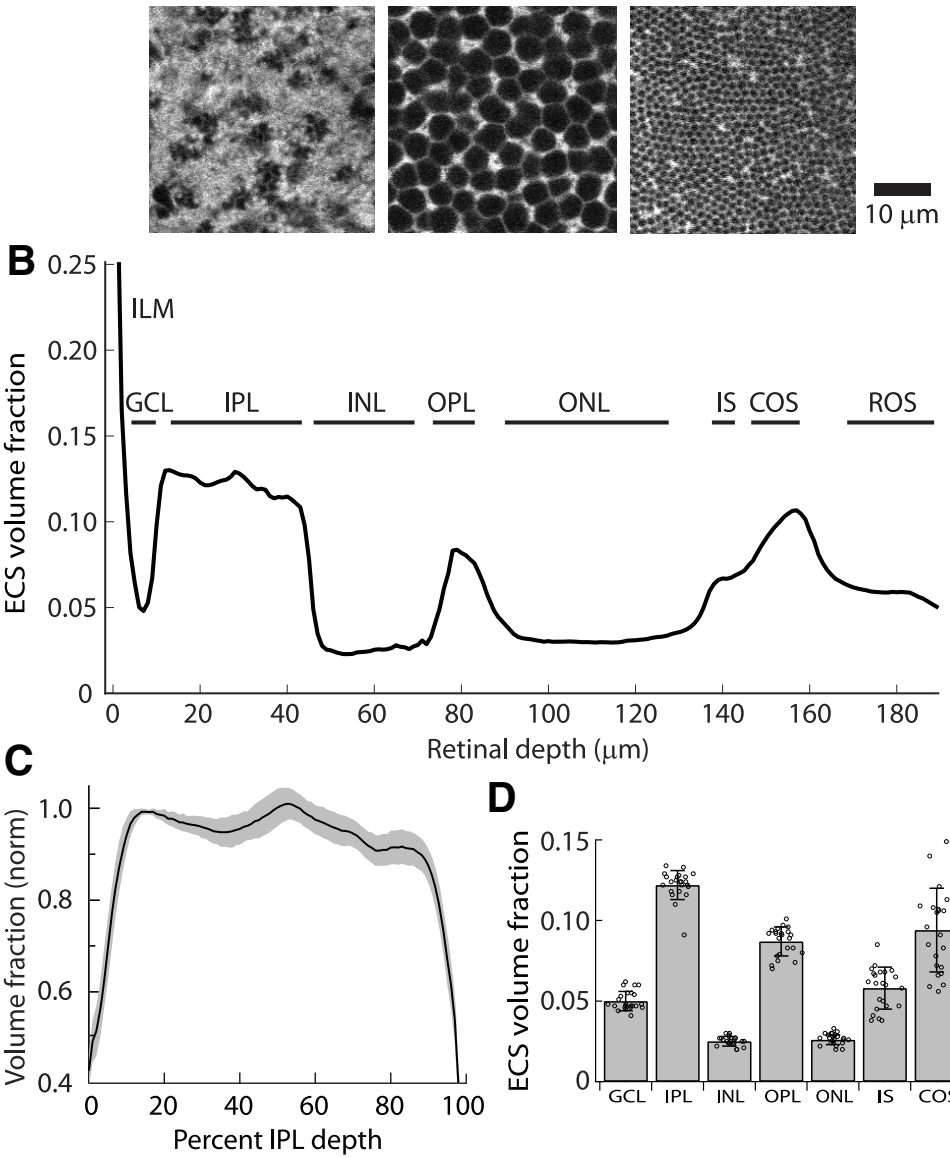

D

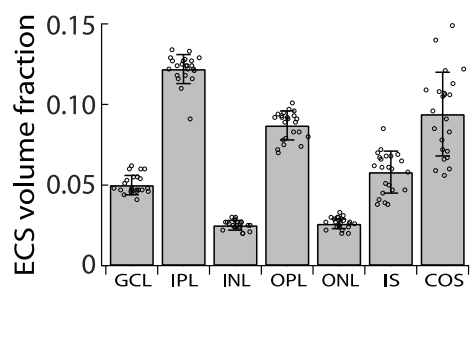

Figure 2. ECS $\alpha$ in different layers of the adult retina. $\boldsymbol{A}, 2 \mathrm{P}$ microscopy images of calcein fluorescence in the outer segments. C, Mean (black line) \pm SD (gray shading) ECS $\alpha$ across the depth of the IPL (normalized to the boundaries; $0 \%$ is $\mathrm{GCL} / \mathrm{IPL}$ border; $n=21)$. $D$, Summary of $\alpha$ in different retinal layers. Bars with error bars retina were as follows: $\mathrm{GCL}, 0.050 \pm 0.006 ; \mathrm{IPL}, 0.122 \pm 0.009 ; \mathrm{INL}, 0.025 \pm 0.003 ; 0 \mathrm{PL}, 0.087 \pm 0.009 ; 0 \mathrm{NL}$, $0.026 \pm 0.003$; photoreceptor inner segment layer, $0.058 \pm 0.013$.

matching simulated data to experimental results and equaled $1.1 \times 10^{-6}$ $\mathrm{cm}^{2} / \mathrm{s}$. For simplicity, $D_{i}$ was assumed to be the same in all retina layers and beyond the boundaries of the retina. A time step of $1 \mu \mathrm{s}$ was used to calculate diffusion in the simulation. This resulted in a mean linear diffusion step length of $12 \mathrm{~nm}$, smaller than the ECS width (Tao and Nicholson, 2004). Particles that reached the boundaries of the model retina continued to diffuse in the space beyond the retina, which had an ECS $\alpha=1.0$. Simulation plots were smoothed with a 0.5 s moving average window.

\section{Experimental design and statistical analysis}

Comparisons among groups were determined using one-way ANOVA followed by Tukey-Kramer multiple-comparisons post hoc tests. Differences were considered significantly different at $p<0.05$. Data are shown as the mean $\pm \mathrm{SD}$. In all cases, $n$ values represent the number of retinas. Statistics were performed using MATLAB (2016b). Results from male and female mice were pooled as there were no statistically significant differences between the two sexes. 


\section{Results}

\section{Visualizing ECS $\alpha$ and cellular morphology across different} retinal layers

We have developed an optical technique for visualizing and accurately measuring $\alpha$ in different retinal layers with high spatial resolution. The retina is superfused with an extracellular solution containing the fluorescent dye calcein. This polar, membrane-impermeant dye (Zhang and Verkman, 2010) rapidly diffuses into the interstitial spaces between retinal cells, filling all extracellular spaces. $2 \mathrm{P}$ microscopy images of the dye within the retina (Fig. 1C) show the ECS brightly labeled with intracellular spaces dark. ECS $\alpha$ within a retinal layer is determined by measuring the spatially integrated dye fluorescence intensity within that layer and comparing it with the dye fluorescence in the superfusate above the retina (Fig. $1 B$ ). Optical sections captured by the $2 \mathrm{P}$ microscope were thin $(z$-plane FWHM $=1.40 \mu \mathrm{m})$, ensuring that measurements accurately reflected ECS $\alpha$ within a single retinal layer.

It is evident from vertical reconstructions of fluorescently labeled extracellular space that the structure of the ECS varies across the retina (Fig. $1 F$ ). The retinal layers are clearly visible, and some large spaces between cellular structures can be resolved. For example, there are prominent extracellular spaces surrounding cone outer segments (Fig. $1 G$ ). However, a substantial fraction of ECS structures, particularly in the synaptic layers, are likely below the resolution of $2 \mathrm{P}$ fluorescence microscopy. Super-resolution light microscopy of live tissue slices as well as electron microscopy of specimens in which ECS is preserved using specialized fixation conditions, has revealed that brain ECS has a highly heterogeneous structure, with interstitial spaces between cells ranging from $\sim 0.05$ to $1 \mu \mathrm{m}$ (Van Harreveld et al., 1965; Korogod et al., 2015; Godin et al., 2017; Tønnesen et al., 2018). However, it is not necessary (or possible) to fully resolve this fine-scale structure of the ECS to measure $\alpha$ using the technique we describe here. Extracellular compartments that are below the diffraction limit of our 2P microscope will still contribute a fluorescence signal that is proportional to the extracellular volume. It is only necessary to obtain an average fluorescence intensity measurement over a region of retina to determine ECS $\alpha$ at a given retinal depth.

This point is illustrated by examination of the cone outer segments, which can be easily differentiated from rod outer segments in vertical reconstructions of the retina (Fig. 1G). In addition to their obvious difference in morphology, cone outer segments are of intermediate brightness compared with the intracellular compartment of rod outer segments and extracellular compartments. This is because cone outer segments are composed of stacks of membranous discs that remain continuous with the plasma membrane and thus are separated by narrow extracellular spaces that are labeled by calcein. Rod outer segment discs, in contrast, are surrounded by a plasma membrane that seals the discs off from the ECS (Carter-Dawson and Lavail, 1979).

\section{Measurement of ECS $\alpha$ across different retinal layers}

We used the calcein fluorescence technique to measure ECS $\alpha$ in the different layers of the mature [postnatal day 45 (P45) to P80] mouse retina. Images of ECS at different depths in the retina were acquired at $1 \mu \mathrm{m}$ intervals, beginning above the vitreal surface of the retina and focusing down through the retina into the rod outer segment layer. In these experiments, measurements were made on isolated flat-mounted retinas in the inverted imaging configuration (see Materials and Methods) to ensure that retinal layers were orthogonal to the imaging axis and to minimize the amount of labeled perfusate solution above the retina.

Figure $2 A$ shows example images of extracellular calcein fluorescence in different retinal layers. The large somata of ganglion cells and displaced amacrine cells in the GCL as well as the smaller somata of horizontal, bipolar, amacrine, and Müller cells in the INL are seen as dark circular profiles. The inner and outer plexiform layers have a reticulated appearance and brighter calcein labeling. A plot of ECS $\alpha$ versus depth for an example retina is shown in Figure $2 B$. Several features are apparent, (1) there are large differences in ECS $\alpha$ between the different retinal layers, with greater ECS $\alpha$ in the synaptic versus somatic layers (Fig. $2 B$, $D)$; (2) the transitions in $\alpha$ between layers are sharp, occurring within a few micrometers; and (3) there are subtle variations in ECS $\alpha$ within the IPL; there is a small, but consistent rise in $\alpha$ at $\sim 50 \%$ depth within the IPL, which is near the transition between $\mathrm{ON}$ and OFF response sublaminae (sublamina 3; Fig. 2B,C). These measurements indicate structural differences of the ECS across different retinal layers that may strongly shape diffusion within the retina as a whole.

\section{ECS $\alpha$ during early development}

Previous studies using the real-time iontophoresis method demonstrated that ECS $\alpha$ in the brain is substantially larger early in development than in the adult (Lehmenkühler et al., 1993; Vorisek and Sykova, 1997). We measured ECS $\alpha$ in the retina during early postnatal development, at P0, P7, and P14 and found that in both the GCL and the INL, spaces between cell bodies were substantially wider in young retinas than in older retinas (Fig. 3A). In accord with these wider spaces, ECS $\alpha$ in the GCL and the INL was larger in young retinas than in the mature retina (Fig. 3B,C).

Profiles of ECS $\alpha$ versus retinal depth clearly show the progression of retinal development (Fig. $3 B$ ). The overall thickness of the retina decreases as retinal development progresses (Dai et al., 2016). This is evident in Figure $3 B$, which shows a leftward shift in the GCL, IPL, and OPL during development (the OPL is not present at P0). In addition, ECS $\alpha$ in the GCL, IPL, and INL decreases with development. Early in development, neuronal somata can be seen within the IPL (Fig. $3 A$ ). These somata were excluded when determining ECS $\alpha$ in the IPL.

\section{Measurement of ECS dynamics}

We next sought to understand whether light-driven neuronal activity can evoke changes in ECS $\alpha$ in the mature retina. We first assessed the temporal resolution of our approach for detecting changes in ECS $\alpha$. A key assumption of our optical method is that calcein concentration is the same throughout the retina and superfusate. If $\alpha$ changes faster than the dye concentration can equilibrate, then fluorescence intensity will not accurately reflect ECS $\alpha$. For instance, a decrease in $\alpha$ that occurs faster than the redistribution of dye molecules will result in an unchanged fluorescence signal until the dye re-equilibrates. We therefore used a combination of experimental and modeling techniques to examine calcein diffusion within the retina to estimate how long it takes for calcein concentration to re-equilibrate following a change in ECS $\alpha$.

We first measured calcein diffusion following iontophoretic ejection of the dye into the middle of the IPL. Calcein was ejected continuously from a glass micropipette for $120 \mathrm{~s}$. Diffusion of the dye was determined by imaging calcein fluorescence with $2 \mathrm{P}$ 
microscopy in the IPL, the INL, and the ONL. In each of these layers, fluorescence intensity was measured in arc-shaped ROIs at a lateral distance of $75 \mu \mathrm{m}$ from the ejection pipette tip (Fig. $4 A, B$ ). Calcein concentration within the ROIs increased as the dye diffused away from the ejection pipette (Fig. 4D).

We next simulated the experimentally measured diffusion of calcein using a 3D retinal model that included different retinal layers (GCL, IPL, INL, OPL, and ONL) with ECS $\alpha$ values for each layer taken from the measurements described above. The iontophoresis experiments were modeled by simulating a point source for calcein in the middle of the IPL (Fig. $4 C$, red sphere). The value of an interstitial space diffusion coefficient of calcein $\left(D_{i}\right)$ in the model was adjusted to obtain the best least-squares match between scaled simulation results and experimental data for diffusion within the IPL. Using this approach, $D_{i}$ equaled $1.1 \times 10^{-6} \mathrm{~cm}^{2} / \mathrm{s}(n$ $=6$ experimental trials). Note that $D_{i}$ is not equivalent to the effective diffusion coefficient $\left(D^{*}\right)$ defined previously (Nicholson and Phillips, 1981). $D_{i}$ factors in the slowing of particle diffusion because of interstitial viscosity, dead spaces, and other factors (Nicholson and Hrabětová, 2017), but not increased path length, which is modeled in the simulations. For simplicity, diffusion was modeled using the same value of $D_{i}$ in all retinal layers. As such, the model is not a precise simulation of diffusion as it does not take into account variations in $D_{i}$ in the different retinal layers, which may have different values (Tao and Nicholson, 2004; Nicholson and KamaliZare, 2020). Nevertheless, the simulation results using a single, uniform diffusion coefficient that was derived from fitting the experimental measurements within the IPL matched the experimental data closely in both time course and amplitude, not only for diffusion within the IPL, but also for diffusion from the IPL into the INL and into the ONL (Fig. 4D). Thus, the model provides a reasonable approximation for diffusion within the retina.

We then used the Monte Carlo simulation to model the diffusion of calcein within the retina following a rapid decrease in $\alpha$ within the IPL, simulated by instantaneously raising the concentration of calcein in the ECS. Using the calcein $D_{i}$ value of $1.1 \times 10^{-6} \mathrm{~cm}^{2} / \mathrm{s}$, we found that an instantaneous reduction in $\alpha$ produces an initial increase in calcein concentration in the IPL that returns to the previous baseline value with a time course that closely follows a first-order exponential decay with a time constant of $11.58 \mathrm{~s}$ (Fig. $4 E$ ). These results indicate that our fluorescence imaging method measures ECS $\alpha$ within $10 \%$ of its true value after an equilibration period of $\sim 26.7 \mathrm{~s}$. Changes in $\alpha$ can still be measured
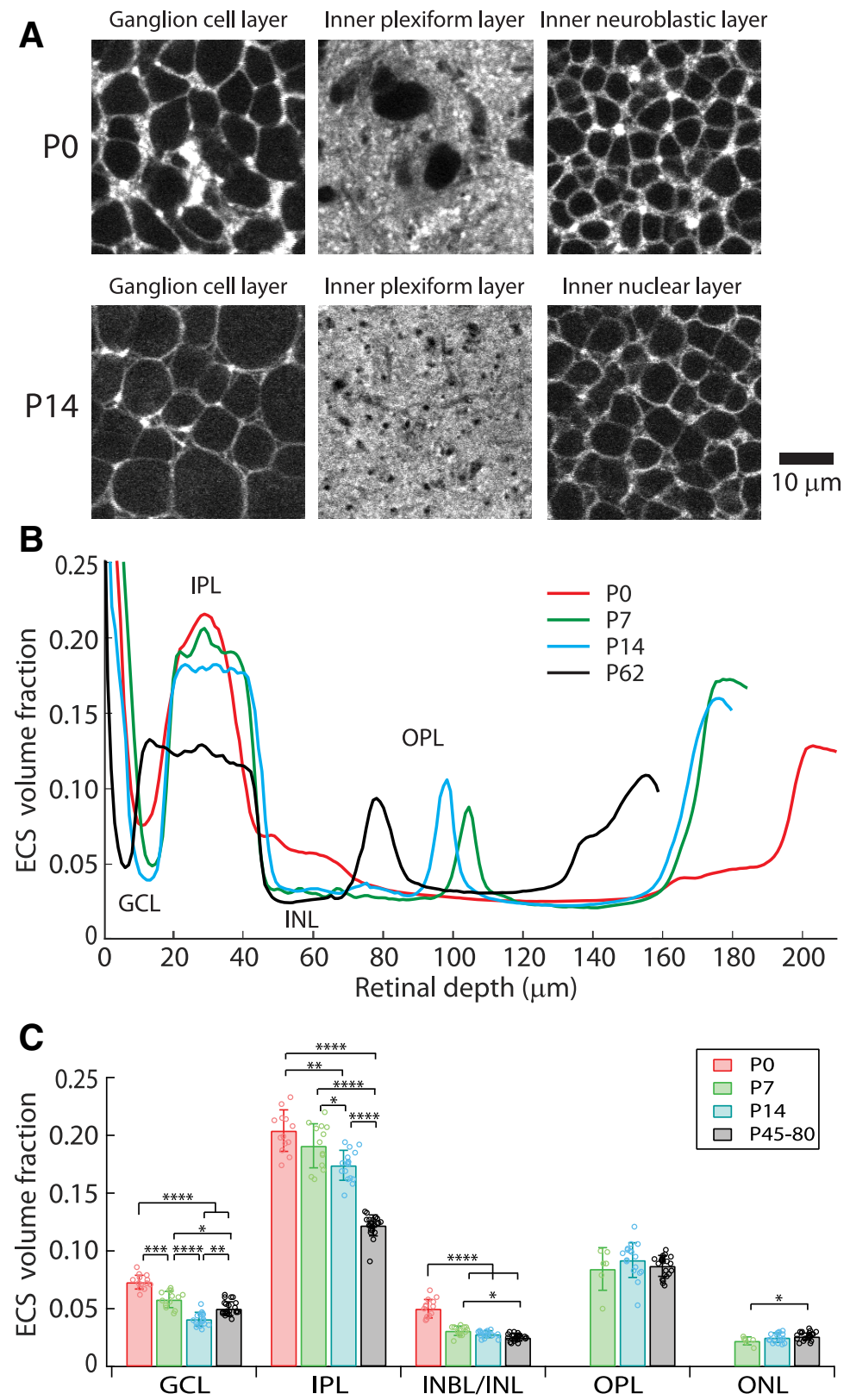

Figure 3. ECS $\alpha$ in different retinal layers during postnatal development. $\boldsymbol{A}, 2 \mathrm{P}$ microscopy images of calcein fluorescence in the GCL, IPL, and the inner neuroblastic layer in a P0 mouse and the GCL, IPL, and INL in a P14 mouse. Extracellular spaces between somata in the cell body layers of the younger retina are larger. Note the somata (dark profiles) in the IPL of the PO animal. $\boldsymbol{B}$, Plots of $\alpha$ as a function of depth beneath the retinal surface in example retinas from P0, P7, P14, and P62 mice. ECS $\alpha$ in the GCL, IPL, and INL progressively shrinks as the retina develops. Note that the OPL is not present at PO and the total thickness of the retina decreases as the retina develops. C, Summary of $\alpha$ in different retinal layers across development. Bars with error bars show the mean $\pm S D(n=13,13,17$, and 23 for P0, P7, P14, and P45 to P80). Open circles show individual measurements. ${ }^{*} p<0.05 ;{ }^{* *} p<0.0001 ;{ }^{* * *} p<10^{-6} ;{ }^{* * *} p<10^{-8}$.

at shorter time periods. Indeed, we observe light-evoked $\alpha$ decreases with a latency as short as $1 \mathrm{~s}$ (see below). However, the measurements at short time intervals will underestimate the true magnitude of rapid $\alpha$ changes as calcein has not had sufficient time to re-equilibrate.

\section{ECS $\alpha$ changes with osmotic challenge}

We next tested whether our fluorescence imaging method is capable of detecting changes in ECS $\alpha$ by using a manipulation known to affect extracellular space, alteration of solution 

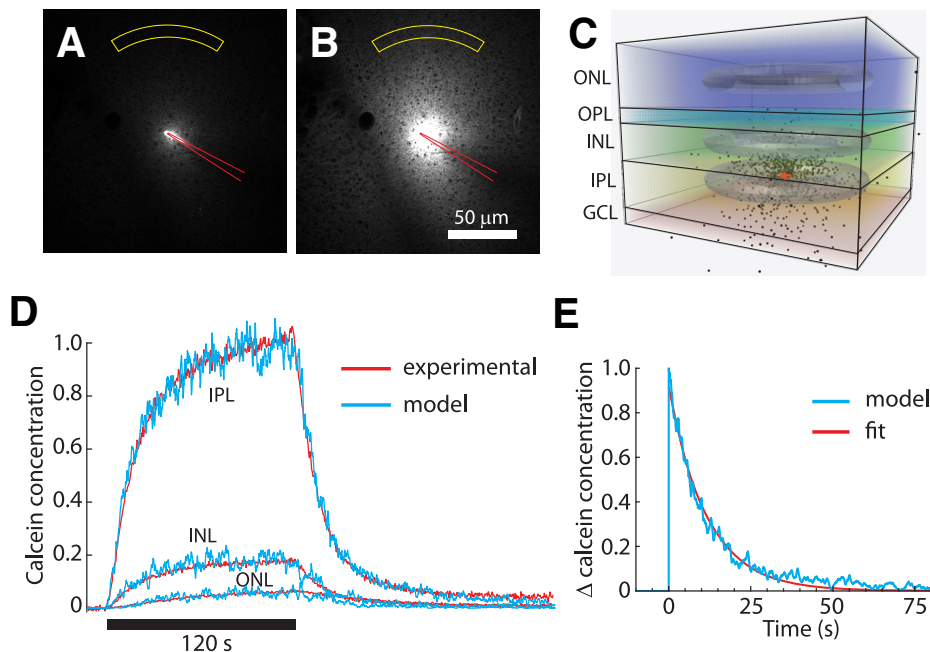

E

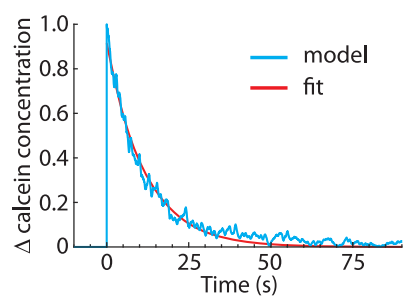

Figure 4. Measurement and computer simulation of calcein diffusion within the retina. $\boldsymbol{A}$, Two-photon microscopy image before iontophoretic ejection of calcein into the IPL. $\boldsymbol{B}$, Same as in $\boldsymbol{A}, 115 \mathrm{~s}$ after the start of the iontophoretic ejection. In $\boldsymbol{A}$ and $\boldsymbol{B}$, calcein fluorescence was measured in the region indicated in yellow; the iontophoretic ejection pipette is outlined in red. C, Schematic of the MCell Monte Carlo diffusion model used to simulate calcein diffusion. The retina is modeled by severa tissue layers, each with its own ECS $\alpha$. Black dots represent calcein molecules. D, Comparison between experimental (red) and model (blue) results for calcein diffusion within the retina. The three experimental traces were obtained in successive trials in a single retina. In the model, calcein was released for $120 \mathrm{~s}$ (black bar) from a point source in the middle of the IPL (red sphere in $\boldsymbol{C}$. Calcein concentration was measured within the three toruses shown in $\boldsymbol{C}$ centered on the calcein source and having a radius of $75 \mu \mathrm{m}$. The toruses are at the mid points of the IPL, INL, and ONL. The diffusion coefficient, $D_{i}$, and the source amplitude of the model were adjusted so that the buildup of calcein concentration in the simulation matched the experimenta trace in the IPL. The simulation matches the experimental results closely, not only in the IPL, but also for diffusion into the INL and the ONL. $\boldsymbol{E}$, Simulation of an $\alpha$ decrease in the IPL. An instantaneous ECS $\alpha$ decrease throughout the entire IPL at time 0 was modeled. The diffusion coefficient determined from the simulation in $\boldsymbol{D}$ was used. The change in calcein concentration in the middle of the IPL is plotted as a function of time. Calcein concentration increases instantaneously with a decrease in $\alpha$ and decays back to baseline with a time course closely following an exponential (red trace; $\tau=11.58 \mathrm{~s}$ ). In $\boldsymbol{D}$ and $\boldsymbol{E}$, the ordinate represents the average calcein concentration per unit volume of tissue.

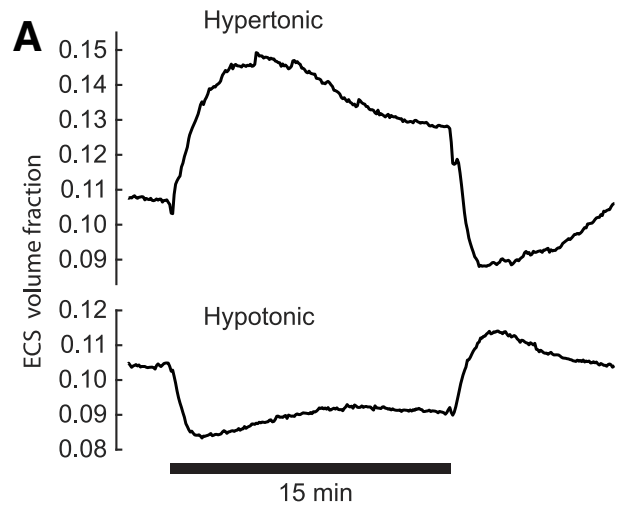

B

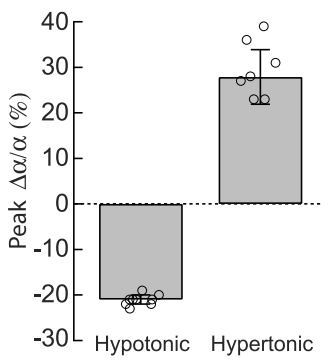

Figure 5. ECS $\alpha$ following hypertonic and hypotonic challenge. A, Example measurements of $\alpha$ in the IPL in response to osmotic challenge. ECS $\alpha$ increases when the retina is superfused with a hypertonic solution (osmolarity increase from 310 to $340 \mathrm{~m} 0 \mathrm{sm} / \mathrm{L}$ ), as retinal cells shrink (top). ECS $\alpha$ decreases when the retina is superfused with a hypotonic solution (osmolarity decrease to $280 \mathrm{~m} 0 \mathrm{sm} / \mathrm{L}$ ), as cells swell (bottom). In both cases, $\alpha$ partially recovers toward baseline during the osmotic challenge and then overshoots following restoration of isotonic solution. ECS $\alpha$ measured in the middle of the IPL. Black bar indicates time course of osmotic challenge. $\boldsymbol{B}$, Summary of peak changes in ECS $\alpha$ in response to altered solution osmolarity. Bars with error bars are the mean $\pm S D$ ( $n=7$ and 9 for hypotonic and hypertonic solutions). Open circles show individual measurements.

osmolarity (Kume-Kick et al., 2002; Hrabetová et al., 2003). We perfused the retina with hypertonic and hypotonic ACSF solutions and measured changes in ECS $\alpha$ in the middle of the IPL. When the perfusate was switched to a hypertonic solution (from $\sim 310$ to $\sim 340 \mathrm{mOsm} / \mathrm{L}$ ), ECS $\alpha$ increased by $28 \%$, from $0.111 \pm 0.006$ to $0.142 \pm 0.050(n=7$; Fig. 5$)$. This $\alpha$ increase reflects a shrinkage of cells in the retina as water moves from intracellular to extracellular compartments. Conversely, when the perfusate was switched to a hypotonic solution $(280 \mathrm{mOsm} / \mathrm{L})$, ECS $\alpha$ decreased by $21 \%$, from $0.105 \pm 0.010$ to $0.083 \pm 0.005$ $(n=9)$. Both the increases and decreases in ECS $\alpha$ partially recovered with continued exposure to the hypertonic and hypotonic solutions, and ECS $\alpha$ overshot baseline levels when isotonic solution was restored.

\section{Light-evoked changes in ECS $\alpha$}

After establishing that our fluorescence imaging method can be used to measure dynamic changes in extracellular space, we tested whether visual stimulation can alter ECS $\alpha$ in the retina. We used a flickering spot $(5 \mathrm{~Hz}$, square-wave modulation, $50 \%$ duty cycle) that was centered on the imaged region for all experiments. Visual stimulation produced changes in ECS $\alpha$ in all retinal layers (Fig. 6A). In most layers, light evoked an ECS $\alpha$ decrease. This change was largest in the IPL (Fig. 6B,C).

We characterized the light-evoked $\alpha$ decrease in the IPL in more detail. The decrease, measured in the middle of the IPL, was rapid for the largest responses. Decreases in ECS $\alpha$ occurred faster than the temporal resolution of our imaging protocol, which was $900 \mathrm{~ms}$. The $\alpha$ decrease was substantial for bright stimuli, equaling $-10.2 \pm 2.5 \%$ of baseline $\alpha$ $(n=15$; spot size, $600 \mu \mathrm{m})$. The magnitude of the decrease in $\alpha$ was proportional to the stimulus intensity (Fig. 7A, $B)$. As discussed above, the measured magnitude of the light-evoked $\alpha$ decrease may underestimate the true magnitude of the decrease at short $(<30 \mathrm{~s})$ time scales.

The magnitude of the light-evoked $\alpha$ decrease in the IPL also varied with stimulus spot diameter over a range of 25$600 \mu \mathrm{m}$ (Fig. 7C). The $\alpha$ decrease was largest for a spot diameter of $100 \mu \mathrm{m}$, which may reflect the center-surround receptive field properties of the neurons (Franke et al., 2017) driving the $\alpha$ decreases. For the brightest stimuli producing large ECS $\alpha$ decreases, there was an overshoot of $\alpha$ at light offset, with a transient increase in ECS $\alpha$ (Fig. 6, IPL trace; Fig. $7 A$, top two traces).

\section{Discussion}

We have developed a method for measuring ECS $\alpha$ that relies on imaging a membrane-impermeant fluorescent dye that is restricted to the extracellular space. The fluorescence imaging 
method can accurately measure ECS $\alpha$ with high spatial resolution in the layered, anisotropic tissue of the retina. It can also measure changes in $\alpha$ evoked by neuronal activity. Although the temporal resolution for quantitative determination of $\alpha$ is limited by the speed of diffusion of calcein within the tissue, $\alpha$ variations with latencies as short as $1 \mathrm{~s}$ were observed.

Our fluorescence technique is derived from previously published methods. Zhang and Verkman (2010) determined ECS $\alpha$ in brain slices by measuring the intensity of a fluorescent dye within the ECS using a microfiber inserted into the tissue. This nonimaging technique has the disadvantage that the microfiber may perturb the geometry of the ECS that is being measured. Tønnesen et al. (2018) measured the brightness of a fluorescent dye in the ECS using superresolution stimulated emission depletion (STED) microscopy in brain slices. A disadvantage of their technique, however, is that STED microscopy does not permit high-quality imaging deeper than $50 \mu \mathrm{m}$ beneath the tissue surface. Further, STED microscopy uses very high intensities of light at wavelengths that strongly excite retinal photoreceptors. The technique we have developed is relatively noninvasive, can image tissue throughout the depth of the retina $(>150 \mu \mathrm{m})$, and allows measurements in tissue with preserved visual sensitivity.

\section{$\mathrm{ECS} \alpha$ in different retinal layers}

To our knowledge, our results represent the first measurements of absolute ECS $\alpha$ values in the retina. They show that $\alpha$ varies dramatically in different retinal layers, from 0.025 in the INL to 0.122 in the IPL. The transitions in $\alpha$ between layers are sharp, changing in just a few micrometers of retinal depth. The relatively smaller ECS $\alpha$ of the INL likely forms a significant diffusion barrier for molecules to move from outer to inner retina and vice versa. ECS $\alpha$ can also vary within layers. A localized rise in $\alpha$ was consistently observed in the middle of the IPL. The variations within the IPL may indicate different patterns and densities of processes and synapses within the different IPL sublaminae (Helmstaedter et al., 2013).

The values of retinal ECS $\alpha$ measured with our fluorescence imaging method, 0.12 in the IPL, are smaller than $\alpha$ values in the cerebral cortex obtained with the real-time iontophoresis method, which are $\sim 0.20$ (Syková and Nicholson, 2008). However, $\alpha$ in the stratum pyramidale of hippocampal slices, a cell body layer, is lower, equaling 0.127 (Saghyan et al., 2012). In addition, real-time iontophoresis measurements are typically made in anesthetized in vivo preparations or in brain slices. More recent real-time iontophoresis measurements in the in vivo, awake rodent cortex yield an ECS $\alpha$ of $\sim 0.14$ (Xie et al., 2013; Sherpa et al., 2016). This discrepancy in brain $\alpha$ values is likely due to a reduction in noradrenergic input to the cortex in the anesthetized and ex vivo preparations (Sherpa et al., 2016).

Although retinal ECS $\alpha$ has not been measured directly before, estimates of retinal $\alpha$ have been made based on transretinal resistance measurements. These studies in frog retinas yielded estimates of 0.07 (Newman and Odette, 1984) and 0.063 (Karwoski et al., 1985a) for the average ECS $\alpha$ across the entire retina. Using the fluorescence measurement technique, we found that the average value of $\alpha$ across the entire mouse retina,

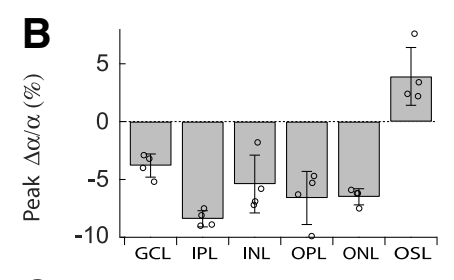

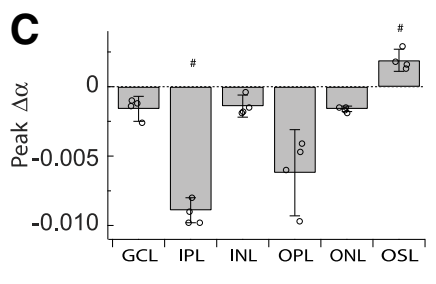

GCL IPL INL OPL ONL OSL

layers of an example retina during light stimulation. The vertical scales of the traces have been adjusted to represent the percentage of changes in $\alpha$, relative to the local baseline $\alpha$. Black bar indicates the stimulus time course: $5 \mathrm{~Hz}$ flickering spot, $100 \mu \mathrm{m}$ diameter, $1.8 \times 10^{4} \mathrm{P} /(\mathrm{S}$ cone $\times \mathrm{s})$. $\boldsymbol{B}$, Summary of peak light-evoked changes in $\alpha$ in different retinal layers, normalized to local baseline $\alpha$ values. Bars with error bars are the mean $\pm \mathrm{SD}(n=4)$. Open circles show individual measurements. $\boldsymbol{C}$, Same as in $\boldsymbol{B}$, but showing absolute changes in ECS $\alpha$. \# indicates groups that are significantly $(p<0.05)$ different from all other groups.
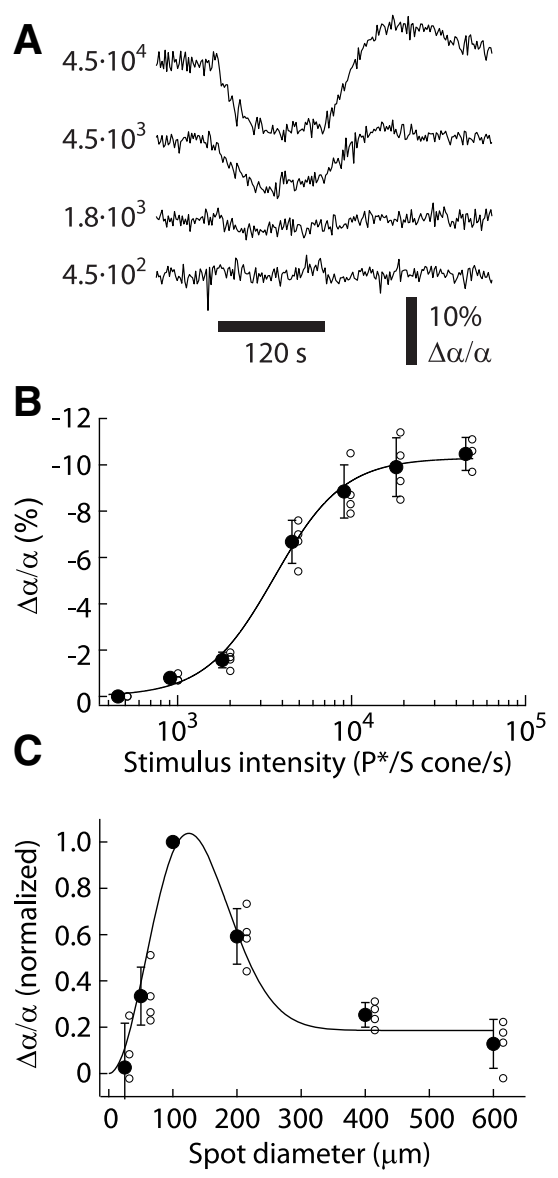

Figure 7. Stimulus intensity and size-dependence of ECS $\alpha$ responses in the IPL. $\boldsymbol{A}, \mathrm{ECS} \alpha$ decreases to stimuli of different intensities, expressed as $P^{*} /(S$ cone $\times s)$. $\boldsymbol{B}$, Intensity-response relation of the peak light-evoked $\alpha$ decrease. Filled circles and error bars are the mean \pm SD $(n=4)$. Open circles show individual trials. Solid curve is the Hill equation fit to mean data points [half-maximal response stimulus intensity of $3.6 \times 10^{3} \mathrm{P} /(\mathrm{S}$ cone $\times \mathrm{s})$ ]. Stimulus, $5 \mathrm{~Hz}$ flickering spot, $100 \mu \mathrm{m}$ diameter. $\boldsymbol{C}$, Light-evoked $\alpha$ decrease as a function of stimulus size. Response amplitudes have been normalized to the response for a $100-\mu \mathrm{m}$-diameter spot. Stimulus, $5 \mathrm{~Hz}$ flickering spot with diameters ranging from 25 to $600 \mu \mathrm{m}, 1.8 \times 10^{4} \mathrm{P} /(\mathrm{S}$ cone $\times \mathrm{s})$ stimulus intensity. Filled circles and error bars are the mean \pm SD $(n=4)$. Open circles show individual trials. Solid curve is the difference-of-Gaussians fit to mean data points (center diameter, $116 \mu \mathrm{m}$; surround diameter, $124 \mu \mathrm{m})$. 
calculated by weighting $\alpha$ in each layer by the thickness of the layer, was $0.061 \pm 0.005 \quad(n=22)$. Resistance measurements also indicated that $\alpha$ varied widely in different retinal layers (Karwoski et al., 1985a), as we observed.

\section{ECS in early development}

Previous studies using the real-time iontophoresis method have demonstrated that ECS $\alpha$ in the brain is substantially larger early in development than in the adult (Lehmenkühler et al., 1993; Vorisek and Sykova, 1997). Our results show that ECS $\alpha$ is larger in the developing retina as well. At P0, ECS $\alpha$ is $67 \%$ larger in the IPL than in the adult, while $\alpha$ in the INL is $100 \%$ larger. As the retina develops, $\alpha$ decreases in these and other layers. The extracellular matrix present in the developing CNS (Long and Huttner, 2019) may help to support these large extracellular spaces.

These changes in ECS $\alpha$ may influence the generation of spontaneous retinal waves that occur in the developing retina (Blankenship et al., 2009; Ford et al., 2012). These waves of RGC activity are essential for the formation of retinotopic maps in the brain (Arroyo and Feller, 2016). They first appear at embryonic day 17 and last until $\sim$ P14 in the mouse (Blankenship and Feller, 2010). The waves are generated by the release of ACh and glutamate from starburst amacrine cells and bipolar cells, respectively. Signaling between starburst amacrine cells is mediated by diffusion of ACh through the ECS rather than by conventional cholinergic synapses (Ford et al., 2012). Similarly, glutamate transmission in early development is mediated by volume transmission (Blankenship et al., 2009). The value of ECS $\alpha$ will influence the concentration and diffusion of $\mathrm{ACh}$ and glutamate throughout the developing retina.

\section{Light-evoked decrease in ECS $\alpha$}

Previous studies in the brain have demonstrated that electrically evoked neuronal activity (Svoboda and Syková, 1991; ProkopováKubinová and Syková, 2000), spreading depression (Mazel et al., 2002), and epileptiform activity (Slais et al., 2008) result in decreases in ECS $\alpha$. It has not been clear, however, whether more modest changes in neuronal activity evoked by physiological stimuli can also drive changes in ECS $\alpha$. Our results demonstrate that light stimulation can evoke large, rapid decreases in ECS $\alpha$. Flickering spot stimulation leads to $\alpha$ decreases as large as $10 \%$ in the IPL. The decreases develop rapidly, with latencies of $<1 \mathrm{~s}$. These light-evoked decreases demonstrate that a physiological stimulus can evoke rapid changes in ECS $\alpha$.

The light-evoked $\alpha$ decreases are likely generated by the swelling of neurons and/or glial cells. Previous studies have indicated that ECS $\alpha$ decreases evoked by electrical stimulation of neurons are due primarily to swelling of astrocytes, the principal glial cell in the brain (Kofuji and Newman, 2004; Larsen and MacAulay, 2017). Swelling could possibly be mediated by the influx of $\mathrm{K}^{+}$ into astrocytes via inwardly rectifying $\mathrm{K}^{+}$channels or the $\mathrm{Na}^{+}$/ $\mathrm{K}^{+} / 2 \mathrm{Cl}^{-}$cotransporter. Recent work, however, demonstrates that neither inwardly rectifying $\mathrm{K}^{+}$channels (Haj-Yasein et al., 2011; Larsen et al., 2014) nor the $\mathrm{Na}^{+} / \mathrm{K}^{+} / 2 \mathrm{Cl}^{-}$cotransporter (Larsen et al., 2014) contribute significantly to activity-dependent $\alpha$ decreases in brain slices. Rather, it has been proposed that astrocyte swelling and ECS shrinkage is generated by the $\mathrm{Na}^{+}$/ $\mathrm{HCO}_{3}^{-}$cotransporter or the monocarboxylate cotransporter (Larsen and MacAulay, 2017). Both of these transporters are expressed in Müller cells, the principal macroglial cell of the retina (Newman and Reichenbach, 1996) and one or both of these systems could be responsible for the observed light-evoked $\alpha$ decreases. It is interesting to note that the light-evoked $\alpha$ decrease we observed in the IPL has a time course similar to the previously described light-evoked increase in ECS $\mathrm{K}^{+}$concentration, which is generated by neuronal depolarization (Steinberg et al., 1980; Karwoski et al., 1985b). The two could be causally related. An increase in ECS $\mathrm{K}^{+}$would depolarize Müller cells, leading to an influx of $\mathrm{Na}^{+}$and $\mathrm{HCO}_{3}^{-}$through the electrogenic $\mathrm{Na}^{+} / \mathrm{HCO}_{3}^{-}$cotransporter (Newman, 1991), leading to cell swelling.

Light adaptation has previously been shown to increase ECS $\alpha$ in the photoreceptor layer of the retina ( $\mathrm{Li}$ et al., 1994), a response we observed as well (Fig. 6). This $\alpha$ increase is generated by ion fluxes across the retinal pigment epithelium, a mechanism that differs from the light-evoked $\alpha$ decreases we observe in the inner retina.

The light-evoked variations in ECS $\alpha$ we observed could have important influences on synaptic transmission in the retina. A decrease in ECS $\alpha$ may reduce the diffusion of neurotransmitter away from the synaptic cleft following its release from the presynaptic terminal and thus result in reduced activation of extrasynaptic receptors. This could be particularly important for glutamatergic synapses in the inner retina, where RGCs with dendrites in the ON laminae of the IPL express NMDA receptors that are primarily localized to perisynaptic regions outside of the synaptic cleft (Chen and Diamond, 2002; Zhang and Diamond, 2006, 2009). Volume transmission of transmitters to distant synapses would also be affected by changes in $\alpha$. This may play a role in circuit adaptation in the retina, which is mediated, in part, by volume transmission of dopamine released from amacrine cells (Witkovsky, 2004).

\section{Conclusion}

We have developed a fluorescence imaging method for measuring ECS $\alpha$ in the retina. Using this technique, we demonstrate that $\alpha$ varies substantially in the different layers of the retina and at different developmental times. We also find that light-evoked neuronal activity can evoke large, rapid decreases in $\alpha$. These light-driven changes in ECS $\alpha$ may regulate synaptic transmission in retinal circuits and may represent a mechanism of synaptic modulation throughout the CNS.

\section{References}

Arroyo DA, Feller MB (2016) Spatiotemporal features of retinal waves instruct the wiring of the visual circuitry. Front Neural Circuits 10:54.

Blankenship AG, Feller MB (2010) Mechanisms underlying spontaneous patterned activity in developing neural circuits. Nat Rev Neurosci 11:18-29.

Blankenship AG, Ford KJ, Johnson J, Seal RP, Edwards RH, Copenhagen DR, Feller MB (2009) Synaptic and extrasynaptic factors governing glutamatergic retinal waves. Neuron 62:230-241.

Carter-Dawson LD, Lavail MM (1979) Rods and cones in the mouse retina. I. Structural analysis using light and electron microscopy. J Comp Neurol 188:245-262.

Chen S, Diamond JS (2002) Synaptically released glutamate activates extrasynaptic NMDA receptors on cells in the ganglion cell layer of rat retina. J Neurosci 22:2165-2173

Dai X, Zhang H, Han J, He Y, Zhang Y, Qi Y, Pang JJ (2016) Effects of subretinal gene transfer at different time points in a mouse model of retinal degeneration. PLoS One 11:e0156542.

Dunn AK, Wallace VP, Coleno M, Berns MW, Tromberg BJ (2000) Influence of optical properties on two-photon fluorescence imaging in turbid samples. Appl Opt 39:1194-1201.

Ford KJ, Félix AL, Feller MB (2012) Cellular mechanisms underlying spatiotemporal features of cholinergic retinal waves. J Neurosci 32:850-863.

Franke K, Berens P, Schubert T, Bethge M, Euler T, Baden T (2017) Inhibition decorrelates visual feature representations in the inner retina. Nature 542:439-444. 
Godin AG, Varela JA, Gao Z, Danné N, Dupuis JP, Lounis B, Groc L, Cognet L (2017) Single-nanotube tracking reveals the nanoscale organization of the extracellular space in the live brain. Nat Nanotechnol 12:238-243.

Haj-Yasein NN, Jensen V, Vindedal GF, Gundersen GA, Klungland A, Ottersen OP, Hvalby Ø, Nagelhus EA (2011) Evidence that compromised $\mathrm{K}^{+}$spatial buffering contributes to the epileptogenic effect of mutations in the human Kir4.1 gene (KCNJ10). Glia 59:1635-1642.

Helmstaedter M, Briggman KL, Turaga SC, Jain V, Seung HS, Denk W (2013) Connectomic reconstruction of the inner plexiform layer in the mouse retina. Nature 500:168-174.

Hrabetová S, Hrabe J, Nicholson C (2003) Dead-space microdomains hinder extracellular diffusion in rat neocortex during ischemia. J Neurosci 23:8351-8359.

Karwoski CJ, Frambach DA, Proenza LM (1985a) Laminar profile of resistivity in frog retina. J Neurophysiol 54:1607-1619.

Karwoski CJ, Newman EA, Shimazaki H, Proenza LM (1985b) Light-evoked increases in extracellular $\mathrm{K}^{+}$in the plexiform layers of amphibian retinas. J Gen Physiol 86:189-213.

Kerr RA, Bartol TM, Kaminsky B, Dittrich M, Chang J-CJ, Baden SB, Sejnowski TJ, Stiles JR (2008) Fast Monte Carlo simulation methods for biological reaction-diffusion systems in solution and on surfaces. SIAM J Sci Comput 30:3126-3126.

Kobat D, Durst ME, Nishimura N, Wong AW, Schaffer CB, Xu C (2009) Deep tissue multiphoton microscopy using longer wavelength excitation. Opt Express 17:13354-13364.

Kofuji P, Newman EA (2004) Potassium buffering in the central nervous system. Neuroscience 129:1045-1056.

Kornfield TE, Newman EA (2014) Regulation of blood flow in the retinal trilaminar vascular network. J Neurosci 34:11504-11513.

Korogod N, Petersen CC, Knott GW (2015) Ultrastructural analysis of adult mouse neocortex comparing aldehyde perfusion with cryo fixation. Elife 4: e05793.

Kume-Kick J, Mazel T, Vorisek I, Hrabĕtová S, Tao L, Nicholson C (2002) Independence of extracellular tortuosity and volume fraction during osmotic challenge in rat neocortex. J Physiol 542:515-527.

Larsen BR, MacAulay N (2017) Activity-dependent astrocyte swelling is mediated by $\mathrm{pH}$-regulating mechanisms. Glia 65:1668-1681.

Larsen BR, Assentoft M, Cotrina ML, Hua SZ, Nedergaard M, Kaila K, Voipio J, MacAulay N (2014) Contributions of the $\mathrm{Na}+/ \mathrm{K}+$-ATPase, NKCC1, and Kir4.1 to hippocampal K+ clearance and volume responses. Glia 62:608-622.

Lehmenkühler A, Syková E, Svoboda J, Zilles K, Nicholson C (1993) Extracellular space parameters in the rat neocortex and subcortical white matter during postnatal development determined by diffusion analysis. Neuroscience 55:339-351.

Li JD, Govardovskii VI, Steinberg RH (1994) Light-dependent hydration of the space surrounding photoreceptors in the cat retina. Vis Neurosci 11:743-752.

Long KR, Huttner WB (2019) How the extracellular matrix shapes neural development. Open Biol 9:180216.

Magzoub M, Zhang H, Dix JA, Verkman AS (2009) Extracellular space volume measured by two-color pulsed dye infusion with microfiberoptic fluorescence photodetection. Biophys J 96:2382-2390.

Mazel T, Richter F, Vargová L, Syková E (2002) Changes in extracellular space volume and geometry induced by cortical spreading depression in immature and adult rats. Physiol Res 51:S85-S93.

Naarendorp F, Esdaille TM, Banden SM, Andrews-Labenski J, Gross OP, Pugh EN (2010) Dark light, rod saturation, and the absolute and incremental sensitivity of mouse cone vision. J Neurosci 30:12495-12507.

Newman E, Reichenbach A (1996) The Müller cell: a functional element of the retina. Trends Neurosci 19:307-312.

Newman EA (1991) Sodium-bicarbonate cotransport in retinal Müller (glial) cells of the salamander. J Neurosci 11:3972-3983.

Newman EA, Odette LL (1984) Model of electroretinogram b-wave generation: a test of the $\mathrm{K}^{+}$hypothesis. J Neurophysiol 51:164-182.
Nicholson C, Hrabětová S (2017) Brain extracellular space: the final frontier of neuroscience. Biophys J 113:2133-2142.

Nicholson C, Kamali-Zare P (2020) Reduction of dimensionality in Monte Carlo simulation of diffusion in extracellular space surrounding cubic cells. Neurochem Res 45:42-52.

Nicholson C, Phillips JM (1981) Ion diffusion modified by tortuosity and volume fraction in the extracellular microenvironment of the rat cerebellum. J Physiol 321:225-257.

Prokopová-Kubinová Š, Syková E (2000) Extracellular diffusion parameters in spinal cord and filum terminale of the frog. J Neurosci Res 62:530-538.

Reum T, Olshausen F, Mazel T, Vorísek I, Morgenstern R, Syková E (2002) Diffusion parameters in the striatum of rats with 6-hydroxydopamine-induced lesions and with fetal mesencephalic grafts. J Neurosci Res 70:680-693.

Rice ME, Okada YC, Nicholson C (1993) Anisotropic and heterogeneous diffusion in the turtle cerebellum: implications for volume transmission. J Neurophysiol 70:2035-2044.

Rosenegger DG, Tran CH, LeDue J, Zhou N, Gordon GR (2014) A high performance, cost-effective, open-source microscope for scanning two-photon microscopy that is modular and readily adaptable. PLoS One 9:e110475.

Saghyan A, Lewis DP, Hrabe J, Hrabetova S (2012) Extracellular diffusion in laminar brain structures exemplified by hippocampus. J Neurosci Methods 205:110-118.

Sherpa AD, Xiao F, Joseph N, Aoki C, Hrabetova S (2016) Activation of $\beta$-adrenergic receptors in rat visual cortex expands astrocytic processes and reduces extracellular space volume. Synapse 70:307-316.

Slais K, Vorisek I, Zoremba N, Homola A, Dmytrenko L, Sykova E (2008) Brain metabolism and diffusion in the rat cerebral cortex during pilocarpine-induced status epilepticus. Exp Neurol 209:145-154.

Steinberg RH, Oakley B 2nd, Niemeyer G (1980) Light-evoked changes in $\left[\mathrm{K}^{+}\right]_{\mathrm{o}}$ in retina of intact cat eye. J Neurophysiol 44:897-921.

Svoboda J, Syková E (1991) Extracellular space volume changes in the rat spinal cord produced by nerve stimulation and peripheral injury. Brain Res $560: 216-224$

Syková E, Nicholson C (2008) Diffusion in brain extracellular space. Physiol Rev 88:1277-1340.

Syková E, Vorísek I, Antonova T, Mazel T, Meyer-Luehmann M, Jucker M, Hájek M, Ort M, Or M, Bures J (2005) Changes in extracellular space size and geometry in APP23 transgenic mice: a model of Alzheimer's disease. Proc Natl Acad Sci U S A 102:479-484.

Tao L, Nicholson C (2004) Maximum geometrical hindrance to diffusion in brain extracellular space surrounding uniformly spaced convex cells. J Theor Biol 229:59-68.

Tønnesen J, Inavalli V, Nägerl UV (2018) Super-resolution imaging of the extracellular space in living brain tissue. Cell 172:1108-1121.e15.

Van Harreveld A, Crowell J, Malhotra SK (1965) A study of extracellular space in central nervous tissue by freeze-substitution. J Cell Biol 25:117-137.

Vorisek I, Sykova E (1997) Ischemia-induced changes in the extracellular space diffusion parameters, $\mathrm{K}+$, and $\mathrm{pH}$ in the developing rat cortex and corpus callosum. J Cereb Blood Flow Metab 17:191-203.

Witkovsky P (2004) Dopamine and retinal function. Doc Ophthalmol 108:17-40.

Xie L, Kang H, Xu Q, Chen MJ, Liao Y, Thiyagarajan M, O’Donnell J, Christensen DJ, Nicholson C, Iliff JJ, Takano T, Deane R, Nedergaard M (2013) Sleep drives metabolite clearance from the adult brain. Science 342:373-377

Ying J, Liu F, Alfano RR (2000) Effect of scattering on nonlinear optical scanning microscopy imaging of highly scattering media. Appl Opt 39:509-514.

Zhang H, Verkman AS (2010) Microfiberoptic measurement of extracellular space volume in brain and tumor slices based on fluorescent dye partitioning. Biophys J 99:1284-1291.

Zhang J, Diamond JS (2006) Distinct perisynaptic and synaptic localization of NMDA and AMPA receptors on ganglion cells in rat retina. J Comp Neurol 498:810-820.

Zhang J, Diamond JS (2009) Subunit- and pathway-specific localization of NMDA receptors and scaffolding proteins at ganglion cell synapses in rat retina. J Neurosci 29:4274-4286. 